The Egyptian Journal of Hospital Medicine (April 2019) Vol. 75 (1), Page 1923-1931

\title{
Evaluation of Knowledge, Attitude and Practice of Epistaxis among the General Population of Tabuk City, Saudi Arabia \\ Mohammed Ahmed Alhejaily ${ }^{1}$, Ahmad Awadh Alatawi ${ }^{1}$, Meshal Saleh Alatawi ${ }^{2}$, Hyder Osman Mrighani ${ }^{3}$
}

1- Medical intern, Faculty of Medicine, University of Tabuk, 2- $3^{\text {rd }}$ year Medical student,

Faculty of Medicine, University of Tabuk, 3- MD, Associate Professor of Internal Medicine and Endocrine,

Department of Medicine Faculty of Medicine, University of Tabuk, KSA

Corresponding author: Mohamed Ahmed Alhejaily, E-mail: alhejailymohammed@gmail.com,

Mobile: +966566669605

\begin{abstract}
Background: epistaxis is a fairly common problem, which has been reported to be occurring in about $60 \%$ of the population. It defined as a bleeding from nose or nasal cavity and it is considered as one of the most common emergencies presenting in Ear, Nose and Throat (ENT) Department and Accident and Emergency Department worldwide. Objectives: this study aimed to assess the knowledge, awareness and practice of the general population of Tabuk City, KSA towards epistaxis, its causes, and management.

Materials and Methods: a cross sectional study was conducted on 540 participants of age less than 20 years to 51 and older in Saudi Arabia. Data were collected by using a pre-designed online questionnaire, which was distributed among the general population of Tabuk City, Saudi Arabia. Results: this study included 540 participants, $71.9 \%$ females and $28.1 \%$ males. Most of the participants $(50.4 \%)$ were from 21 to 30 years. Epistaxis was found in $45.2 \%$ of them. Among participants, $34.8 \%$ knew that chronic diseases cause epistaxis, $42.2 \%$ stated that drugs cause epistaxis and $68.9 \%$ knew that excess nose manipulation caused epistaxis. Tilting the head forward was the best position to stop epistaxis which reported by $45.2 \%$ and $42.2 \%$ reported cartilage as a part of nose should be pressed on to stop epistaxis. There were no significant correlations between educational level and different knowledges of the causes and how to deal with epistaxis case ( $p>0.05)$. However, we found that there was a significant correlation with the action should be done if the epistaxis continues $(\mathrm{p}=0.001)$, the best position to stop epistaxis ( $\mathrm{p}=0.02$ ) and nose manipulation as a cause of epistaxis $(\mathrm{p}=0.01)$.

Conclusion: our results showed that high percent of the participants were suffering from nasal bleeding (Epistaxis) in Saudi Arabia. And there was good a knowledge about causes of epistaxis and how to deal with epistaxis cases.
\end{abstract}

Keywords: epistaxis, bleeding, knowledge, awareness, Saudi Arabia

\section{INTRODUCTION}

Epistaxis is acute bleeding from the nasopharynx or the nose. It is one of the most common ENT emergencies faced in the emergency departments worldwide ${ }^{[1]}$. Epistaxis is a common problem and it ranges from mild to severe bleeding, also it is considers as life-threatening rhinological emergency. It acts as a significant workload in accident and emergency and Otolaryngology Departments; it usually causes anxiety for both patients and clinicians ${ }^{[2]}$. Of population, $66.67 \%$ experience epistaxis during their lifetime.

It is common among school children as they are easily injured during different school activity. More than $50 \%$ of children between 6 and 10 years have suffered from at least one episode of epistaxis [3]. Epistaxis reportedly occurs more frequently during the dry, cold winter months. It is thought to occur more frequently in males than in females and there is an increasing incidence with age ${ }^{[4]}$. The nasal bleeding caused by either systemic or local factor, the systemic factors involved coagulopathy, blood disorders, the use of anticoagulant and arterial high blood pressure, while the local factors included upper airway infections, nasal allergies, the introduction of foreign bodies into the nasal cavity, trauma and septal perforation ${ }^{[5]}$. Other causes have been reported in children from nose picking and idiopathic causes, to trauma, medications, nasopharyngeal mass, bacterial nasal colonization and allergic rhinitis and to the less common cause such as ectopic intranasal tooth ${ }^{[6}$, ${ }^{7]}$.Epistaxis can be broadly divided as occurring from anterior or posterior sites. Anterior epistaxis is more frequent at an early age. Its origin can be either arterial (Keisselbach area) or venous (Retrocolumellar vein). As the bleeding point is easily accessible, this type of epistaxis is rarely serious. Posterior epistaxis, on the other hand, is more frequent in the elderly and they may be a major therapeutic problem ${ }^{[4]}$.

In most of the cases, epistaxis usually occurs in an out of the hospital setting. Thus, it is very important for non- health professional people to understand and know some first aid measures for epistaxis. First aid is important to reduce mortality and morbidity of the emergency case ${ }^{[8]}$ especially in the persistent bleeding cases ${ }^{[9]}$. The management of epistaxis is complex and varied and it has undergone significant changes over the years from 
the uncomfortable posterior nasal pack to the newly developed packing devices, indigenous hemostatic agents and endoscopic surgical approaches ${ }^{[10]}$.

This study aimed to assess the knowledge, awareness and practice of the general population of Tabuk City, KSA towards epistaxis, its causes and management.

\section{MATERIALS AND METHODS}

This was a descriptive cross-sectional study conducted on a random sample of the population of Tabuk City, Saudi Arabia. A convenient sample size of 540 with different age groups had participated in this study. Approval from the Research and Ethical Committee was obtained. Incomplete questionnaire was taken as exclusion criteria.

Data Collection: data were collected by using a predesigned online questionnaire, which was distributed among the general population of Tabuk City, Saudi Arabia. This study was conducted during the period from June to October 2017. It was self-administered by participants. The questionnaire included the relevant questions to collect data about:

- Socio-demographic characteristics included age, marital status, educational level and previous exposure to epistaxis.

- Other sections included questions designed for assessment of the level of knowledge, awareness and practice of the study sample towards epistaxis.

Data Analysis: the data were compiled, checked for completeness, and analyzed by using the Statistical Package for Social Sciences (SPSS Inc., Chicago, IL, USA) version 22. Results of descriptive analysis of knowledge and awareness of epistaxis were tabulated. Cross tabulations included Chi-square test were designed to find the correlation between the previous exposure to epistaxis and educational level with other knowledge assessment factors. A $5 \%$ level was chosen as a level of significance in all statistical tests used in the study.

\section{Ethical considerations}

All ethical issues were taken into consideration, including the confidentiality and privacy of participant's data. Participants were informed that participation was completely voluntary and data collection website introduced and explained the research to participants. No names were recorded on the questionnaires and all questionnaire responses kept safe.

\section{RESULTS}

Table 1: showed the socio-demographic characteristics of the studied population and prevalence of epistaxis among them. This study included 540 participants, females represent $71.9 \%$ of them and only $28.1 \%$ were males. Half of participants were from 21 to 30 years, $62.2 \%$ were married and the majority $73.3 \%$ had university education or more. Epistaxis was found in $45.2 \%$ of cases.

Table 2: showed the questions of knowledge of the causes of epistaxis and how to deal with epistaxis cases. About one-third (34.8\%) of cases knew that chronic diseases cause epistaxis, $42.2 \%$ stated that drugs cause epistaxis and $68.9 \%$ knew that excess nose manipulation cause epistaxis. More than half of cases (55.6\%) knew that pressing on the nose stops epistaxis. Regarding to best position to stop epistaxis, $45.2 \%$ stated that tilted the head forward and $36.3 \%$ reported tilted the head back. $61.5 \%$ of cases reported breath from the mouth as usual as a method for breathing during epistaxis. $81.5 \%$ prefer going to the hospital if the epistaxis continue. Although, the majority of cases $89.6 \%$ found that first aid measures are important, $85.9 \%$ of them knew that there was no enough awareness of the public about epistaxis first aid measures. ENT physicians reported as a specialist who can treat epistaxis by $67.4 \%$ of cases.

Table 3: showed the relation between knowledge of causes of epistaxis and how to deal with epistaxis cases and having epistaxis attacks. Our study found that there were no significant correlations between having epistaxis attacks and physician who can treat epistaxis, no enough awareness of the public about epistaxis first aid measures, importance of first aid measures, knowing method of breathing during epistaxis, the action should be done if the epistaxis continues, knowing which part of the nose must be pressed to stop epistaxis, the best position to stop epistaxis and knowing that pressing on the nose can stop epistaxis. Also, there was no relation between it and drugs and chronic diseases as a cause epistaxis $(p>0.05)$. However, we found that there were significant correlations between epistaxis attack and nose manipulation $(\mathrm{p}=0.02)$.

Table 4: showed the relation between knowledge of the causes of epistaxis and how to deal with epistaxis cases and educational level. Our study showed no significant correlations between educational level and different knowledge of the causes and how to deal with epistaxis case ( $p>0.05$ ). However, we found that there was a significant correlation with the action should be done if the epistaxis continues $(\mathrm{p}=0.001)$, the best position to stop epistaxis $(p=0.02)$ and nose manipulation as a cause of epistaxis $(\mathrm{p}=0.01)$. 
Table 1: socio-demographic characteristics of the studied population and prevalence of epistaxis among them $(\mathrm{N}=\mathbf{5 4 0})$

\begin{tabular}{|c|c|c|}
\hline Sex & Frequency & Percent \\
\hline$\bullet \quad$ Female & 388 & 71.9 \\
\hline Male & 152 & 28.1 \\
\hline Age & & \\
\hline$\bullet \quad$ Less than 20 years & 48 & 8.9 \\
\hline From 21 to 30 years & 272 & 50.4 \\
\hline$\bullet \quad$ From 31 to 40 years & 124 & $\mathbf{2 3 . 0}$ \\
\hline$\bullet \quad$ From 41 to 50 years & 44 & 8.1 \\
\hline$\bullet$ From 51 years and older & 52 & 9.6 \\
\hline Marital status & & 33.3 \\
\hline$\bullet \quad$ Single & 180 & 62.2 \\
\hline$\bullet \quad$ Married & 336 & 4.4 \\
\hline Divorced/widow & 24 & 5.9 \\
\hline Educational level & 32 & 20.7 \\
\hline Less than secondary & 112 & 73.3 \\
\hline$\bullet$ secondary & 396 & \\
\hline University or more & & 54.8 \\
\hline Having Epistaxis & 296 & 45.2 \\
\hline$\quad$ No & 244 & \\
\hline$\bullet \quad$ Yes & & \\
\hline
\end{tabular}

Table 2: questions of knowledge of the causes of epistaxis and how to deal with epistaxis cases

\begin{tabular}{|c|c|c|}
\hline Chronic diseases are a risk factor & & \\
\hline - No & 104 & 19.3 \\
\hline - Don't know & 248 & 45.9 \\
\hline - Yes & 188 & 34.8 \\
\hline \multicolumn{3}{|l|}{ Some medication can cause epistaxis } \\
\hline - No & 88 & 16.3 \\
\hline - Don't know & 224 & 41.5 \\
\hline - Yes & 228 & 42.2 \\
\hline \multicolumn{3}{|c|}{ Excess Nose Manipulation can cause Epistaxis } \\
\hline - No & 212 & 17.0 \\
\hline - Don't know & 76 & 14.1 \\
\hline - Yes & 372 & 68.9 \\
\hline \multicolumn{3}{|c|}{ Pressing on the nose can stops epistaxis } \\
\hline - No & 136 & 25.2 \\
\hline - Don't know & 104 & $\mathbf{1 9 . 3}$ \\
\hline - Yes & 300 & 55.6 \\
\hline \multicolumn{3}{|c|}{ What is the best position to stop epistaxis } \\
\hline - Lie down with your feet up & 28 & 5.2 \\
\hline - Tilt the head forward & 244 & 45.2 \\
\hline - Tilt the head back & 196 & 36.3 \\
\hline - I do not know & 72 & 13.3 \\
\hline \multicolumn{3}{|c|}{ What part of the nose compressed during epistaxis } \\
\hline - The lower part (cartilage) & 228 & 42.2 \\
\hline - Upper part (bone) & 180 & 33.3 \\
\hline - I do not know & 132 & 24.4 \\
\hline \multicolumn{3}{|c|}{ Method of breathing during epistaxis } \\
\hline - Breath from the nose as usual & 32 & 5.9 \\
\hline
\end{tabular}


Evaluation of Knowledge, Attitude and Practice of Epistaxis...

\begin{tabular}{|c|c|c|}
\hline \multicolumn{3}{|l|}{ Chronic diseases are a risk factor } \\
\hline - Breath from the mouth as usual & 332 & 61.5 \\
\hline - Don't know & 176 & 32.6 \\
\hline \multicolumn{3}{|c|}{ What should be done if bleeding doesn't stop } \\
\hline - Continue the first aid measures & 68 & 12.6 \\
\hline - Just wait & 32 & 5.9 \\
\hline - Go to the hospital & 440 & 81.5 \\
\hline \multicolumn{3}{|l|}{ Are first aid measures important } \\
\hline - No & 12 & 2.2 \\
\hline - Don't know & 44 & 8.1 \\
\hline - Yes & 484 & 89.6 \\
\hline \multicolumn{3}{|c|}{ There is no enough awareness of the public about epistaxis first aid measures } \\
\hline - No & 24 & 4.4 \\
\hline - Don't know & 52 & 9.6 \\
\hline - Yes & 464 & 85.9 \\
\hline \multicolumn{3}{|c|}{ What is the specialty that can treat epistaxis } \\
\hline - Any physician & 120 & 22.2 \\
\hline - ENT physicians & 364 & 67.4 \\
\hline - Don't know & 56 & $\mathbf{1 0 . 4}$ \\
\hline
\end{tabular}

Table 3: relation between knowledge of the causes of epistaxis and how to deal with epistaxis cases and having epistaxis attacks

\begin{tabular}{|c|c|c|c|c|c|}
\hline \multirow[t]{2}{*}{ Questions } & \multirow[t]{2}{*}{ Responses } & \multicolumn{2}{|c|}{ Have epistaxis attacks } & \multirow{2}{*}{$\begin{array}{c}\text { Total } \\
(\mathrm{N}=540)\end{array}$} & \multirow{2}{*}{ P value } \\
\hline & & Yes $(n=244)$ & No $(n=296)$ & & \\
\hline \multirow{6}{*}{ Who can treat epistaxis } & \multirow{2}{*}{ Any doctor } & 36 & 84 & 120 & \multirow{6}{*}{$\mathbf{0 . 0 8 9}$} \\
\hline & & $14.8 \%$ & $28.4 \%$ & $22.2 \%$ & \\
\hline & \multirow{2}{*}{$\begin{array}{l}\text { Ear, Nose and Throat } \\
\text { Doctor }\end{array}$} & 188 & 176 & 364 & \\
\hline & & $77.0 \%$ & $59.5 \%$ & $67.4 \%$ & \\
\hline & \multirow[t]{2}{*}{ I do not know } & 20 & 36 & 56 & \\
\hline & & $8.2 \%$ & $12.2 \%$ & $10.4 \%$ & \\
\hline \multirow{6}{*}{$\begin{array}{l}\text { There is no enough awareness of } \\
\text { public about epistaxis first aid } \\
\text { measures }\end{array}$} & \multirow[t]{2}{*}{ No } & 4 & 20 & 24 & \multirow[t]{6}{*}{0.305} \\
\hline & & $1.6 \%$ & $6.8 \%$ & $4.4 \%$ & \\
\hline & \multirow[t]{2}{*}{ I do not know } & 28 & 24 & 52 & \\
\hline & & $11.5 \%$ & $8.1 \%$ & $9.6 \%$ & \\
\hline & \multirow[t]{2}{*}{ Yes } & 212 & 252 & 464 & \\
\hline & & $86.9 \%$ & $85.1 \%$ & $85.9 \%$ & \\
\hline \multirow[t]{6}{*}{ Are first aid measures important } & \multirow[t]{2}{*}{ No } & 8 & 4 & 12 & \multirow[t]{6}{*}{0.750} \\
\hline & & $3.3 \%$ & $1.4 \%$ & $2.2 \%$ & \\
\hline & \multirow[t]{2}{*}{ I do not know } & 20 & 24 & 44 & \\
\hline & & $8.2 \%$ & $8.1 \%$ & $8.1 \%$ & \\
\hline & \multirow[t]{2}{*}{ Yes } & 216 & 268 & 484 & \\
\hline & & $88.5 \%$ & $90.5 \%$ & $89.6 \%$ & \\
\hline \multirow{6}{*}{$\begin{array}{l}\text { What should be done if the } \\
\text { epistaxis continue }\end{array}$} & \multirow[t]{2}{*}{ Continue to do first aid } & 36 & 32 & 68 & \multirow[t]{6}{*}{0.743} \\
\hline & & $14.8 \%$ & $10.8 \%$ & $12.6 \%$ & \\
\hline & \multirow{2}{*}{$\begin{array}{l}\text { Wait until the bleeding } \\
\text { stops }\end{array}$} & 16 & 16 & 32 & \\
\hline & & $6.6 \%$ & $5.4 \%$ & $5.9 \%$ & \\
\hline & \multirow[t]{2}{*}{ Transfer to hospital } & 192 & 248 & 440 & \\
\hline & & $78.7 \%$ & $83.8 \%$ & $81.5 \%$ & \\
\hline \multirow{3}{*}{$\begin{array}{l}\text { Method of breathing during } \\
\text { epistaxis }\end{array}$} & \multirow{3}{*}{$\begin{array}{l}\text { Preathe through the nose } \\
\text { til the bleeding stops }\end{array}$} & 8 & 24 & 32 & \multirow[t]{3}{*}{0.327} \\
\hline & & $3.3 \%$ & $8.1 \%$ & $5.9 \%$ & \\
\hline & & 164 & 168 & 332 & \\
\hline
\end{tabular}




\begin{tabular}{|c|c|c|c|c|c|}
\hline \multirow[t]{5}{*}{ Questions } & \multirow[t]{2}{*}{ Responses } & \multicolumn{2}{|c|}{ Have epistaxis attacks } & \multirow{2}{*}{$\begin{array}{c}\text { Total } \\
(\mathrm{N}=540)\end{array}$} & \multirow{2}{*}{ P value } \\
\hline & & Yes $(n=244)$ & No $(n=296)$ & & \\
\hline & $\begin{array}{l}\text { Breathing through the } \\
\text { uth until the bleeding } \\
\text { stands }\end{array}$ & $67.2 \%$ & $56.8 \%$ & $61.5 \%$ & \\
\hline & \multirow[t]{2}{*}{ I do not know } & 72 & 104 & 176 & \\
\hline & & $29.5 \%$ & $35.1 \%$ & $32.6 \%$ & \\
\hline \multirow{6}{*}{$\begin{array}{l}\text { Which part of the nose must be } \\
\text { pressed to stop epistaxis }\end{array}$} & \multirow{2}{*}{$\begin{array}{l}\text { The lower part } \\
\text { (cartilage) }\end{array}$} & 100 & 128 & 228 & \multirow[t]{6}{*}{0.822} \\
\hline & & $41.0 \%$ & $43.2 \%$ & $42.2 \%$ & \\
\hline & \multirow[t]{2}{*}{ Upper part (bone) } & 88 & 92 & 180 & \\
\hline & & $36.1 \%$ & $31.1 \%$ & $33.3 \%$ & \\
\hline & \multirow[t]{2}{*}{ I do not know } & 56 & 76 & 132 & \\
\hline & & $23.0 \%$ & $25.7 \%$ & $24.4 \%$ & \\
\hline \multirow{8}{*}{$\begin{array}{l}\text { What is the best position to stop } \\
\text { epistaxis }\end{array}$} & \multirow{2}{*}{$\begin{array}{l}\text { Lie down with your feet } \\
\text { up }\end{array}$} & 12 & 16 & 28 & \multirow[t]{8}{*}{0.332} \\
\hline & & $4.9 \%$ & $5.4 \%$ & $5.2 \%$ & \\
\hline & \multirow[t]{2}{*}{ Tilt the head forward } & 104 & 140 & 244 & \\
\hline & & $42.6 \%$ & $47.3 \%$ & $45.2 \%$ & \\
\hline & \multirow[t]{2}{*}{ Tilt the head back } & 92 & 104 & 196 & \\
\hline & & $37.7 \%$ & $35.1 \%$ & $36.3 \%$ & \\
\hline & \multirow[t]{2}{*}{ I do not know } & 36 & 36 & 72 & \\
\hline & & $14.8 \%$ & $12.2 \%$ & $13.3 \%$ & \\
\hline \multirow{6}{*}{$\begin{array}{l}\text { Pressing on the nose stops } \\
\text { epistaxis }\end{array}$} & \multirow[t]{2}{*}{ No } & 68 & 68 & 136 & \multirow[t]{6}{*}{0.603} \\
\hline & & $27.9 \%$ & $23.0 \%$ & $25.2 \%$ & \\
\hline & \multirow[t]{2}{*}{ I do not know } & 52 & 52 & 104 & \\
\hline & & $21.3 \%$ & $17.6 \%$ & $19.3 \%$ & \\
\hline & \multirow[t]{2}{*}{ Yes } & 124 & 176 & 300 & \\
\hline & & $\mathbf{5 0 . 8 \%}$ & $\mathbf{5 9 . 5 \%}$ & $55.6 \%$ & \\
\hline \multirow{6}{*}{$\begin{array}{l}\text { xcess Nose Manipulation causes } \\
\text { Epistaxis }\end{array}$} & \multirow[t]{2}{*}{ No } & 32 & 60 & 92 & \multirow[t]{6}{*}{$\mathbf{0 . 0 2 3}$} \\
\hline & & $13.1 \%$ & $20.3 \%$ & $17.0 \%$ & \\
\hline & I do not know & 16 & 60 & 76 & \\
\hline & & $6.6 \%$ & $20.3 \%$ & $14.1 \%$ & \\
\hline & Yeah & 196 & 176 & 372 & \\
\hline & & $80.3 \%$ & $59.5 \%$ & $68.9 \%$ & \\
\hline Drugs cause epistaxis & No & 60 & 28 & 88 & 0.060 \\
\hline & & $24.6 \%$ & $9.5 \%$ & $16.3 \%$ & \\
\hline & I do not know & 92 & 132 & 224 & \\
\hline & & $37.7 \%$ & $44.6 \%$ & $41.5 \%$ & \\
\hline & Yeah & 92 & 136 & 228 & \\
\hline & & $37.7 \%$ & $45.9 \%$ & $42.2 \%$ & \\
\hline Chronic diseases cause epistaxis & No & 48 & 56 & 104 & 0.763 \\
\hline & & $19.7 \%$ & $18.9 \%$ & $19.3 \%$ & \\
\hline & I do not know & 104 & 144 & 248 & \\
\hline & & $42.6 \%$ & $48.6 \%$ & $45.9 \%$ & \\
\hline & Yeah & 92 & 96 & 188 & \\
\hline & & $37.7 \%$ & $32.4 \%$ & $34.8 \%$ & \\
\hline
\end{tabular}


Table 4: relation between knowledge of the causes of epistaxis and how to deal with epistaxis cases and

Educational level

\begin{tabular}{|c|c|c|c|c|c|c|}
\hline \multirow[t]{2}{*}{ Questions } & \multirow[t]{2}{*}{ Responses } & \multicolumn{3}{|c|}{\begin{tabular}{|c|} 
Educational level \\
\end{tabular}} & \multirow{2}{*}{$\begin{array}{r}\text { Total } \\
(\mathrm{N}=\mathbf{5 4 0})\end{array}$} & \multirow{2}{*}{$\begin{array}{c}\mathbf{P} \\
\text { value }\end{array}$} \\
\hline & & $\begin{array}{l}\text { Less than } \\
\text { econdary } \\
(\mathrm{n}=32)\end{array}$ & $\begin{array}{l}\text { Secondary } \\
(n=112)\end{array}$ & $\begin{array}{l}\text { University } \\
\text { or more } \\
(n=396)\end{array}$ & & \\
\hline \multirow[t]{6}{*}{ Who can treat epistaxis } & \multirow[t]{2}{*}{ Any doctor } & 4 & 16 & 100 & 120 & \multirow[t]{6}{*}{0.370} \\
\hline & & $12.5 \%$ & $14.3 \%$ & $25.3 \%$ & $22.2 \%$ & \\
\hline & \multirow{2}{*}{$\begin{array}{c}\text { Ear, Nose and Throat } \\
\text { Doctor }\end{array}$} & 28 & 88 & 248 & 364 & \\
\hline & & $87.5 \%$ & $78.6 \%$ & $62.6 \%$ & $67.4 \%$ & \\
\hline & \multirow[t]{2}{*}{ I do not know } & $\mathbf{0}$ & 8 & 48 & 56 & \\
\hline & & $.0 \%$ & $7.1 \%$ & $12.1 \%$ & $10.4 \%$ & \\
\hline \multirow{6}{*}{$\begin{array}{c}\text { There is no enough } \\
\text { awareness of the public } \\
\text { about epistaxis first aid } \\
\text { measures }\end{array}$} & \multirow[t]{2}{*}{ No } & $\mathbf{0}$ & 8 & 16 & 24 & \multirow[t]{6}{*}{0.890} \\
\hline & & $.0 \%$ & $7.1 \%$ & $\mathbf{4 . 0 \%}$ & $4.4 \%$ & \\
\hline & \multirow[t]{2}{*}{ I do not know } & 4 & 8 & 40 & 52 & \\
\hline & & $12.5 \%$ & $7.1 \%$ & $10.1 \%$ & $9.6 \%$ & \\
\hline & \multirow[t]{2}{*}{ Yeas } & 28 & 96 & 340 & 464 & \\
\hline & & $87.5 \%$ & $85.7 \%$ & $85.9 \%$ & $85.9 \%$ & \\
\hline \multirow{6}{*}{$\begin{array}{l}\text { Are first aid measures } \\
\text { important }\end{array}$} & \multirow[t]{2}{*}{ No } & $\mathbf{0}$ & 4 & 8 & 12 & \multirow[t]{6}{*}{0.314} \\
\hline & & $.0 \%$ & $3.6 \%$ & $2.0 \%$ & $2.2 \%$ & \\
\hline & \multirow[t]{2}{*}{ I do not know } & $\mathbf{0}$ & $\mathbf{0}$ & 44 & 44 & \\
\hline & & $.0 \%$ & $.0 \%$ & $11.1 \%$ & $8.1 \%$ & \\
\hline & \multirow[t]{2}{*}{ Yeas } & 32 & 108 & 344 & 484 & \\
\hline & & $100.0 \%$ & $96.4 \%$ & $86.9 \%$ & $89.6 \%$ & \\
\hline \multirow{6}{*}{$\begin{array}{l}\text { What should be done if } \\
\text { the epistaxis continue }\end{array}$} & \multirow[t]{2}{*}{ Continue to do first aid } & 4 & 40 & 24 & 68 & 0.001 \\
\hline & & $12.5 \%$ & $35.7 \%$ & $6.1 \%$ & $12.6 \%$ & \\
\hline & Wait until the bleeding & 0 & 8 & 24 & 32 & \\
\hline & $\begin{array}{c}\text { stops } \\
\end{array}$ & $.0 \%$ & $7.1 \%$ & $6.1 \%$ & $\mathbf{5 . 9 \%}$ & \\
\hline & Transfer to hospital & 28 & 64 & 348 & 440 & \\
\hline & & $87.5 \%$ & $57.1 \%$ & $87.9 \%$ & $81.5 \%$ & \\
\hline Method of breathing & Breathe through the & $\mathbf{0}$ & 12 & 20 & 32 & 0.762 \\
\hline during epistaxis & $\begin{array}{c}\text { nose until the bleeding } \\
\text { stops } \\
\end{array}$ & $.0 \%$ & $10.7 \%$ & $5.1 \%$ & $5.9 \%$ & \\
\hline & Breathing through the & 20 & 64 & 248 & 332 & \\
\hline & $\begin{array}{l}\text { nouth until the bleeding } \\
\text { stands }\end{array}$ & $62.5 \%$ & $57.1 \%$ & $62.6 \%$ & $61.5 \%$ & \\
\hline & I do not know & 12 & 36 & 128 & 176 & \\
\hline & & $37.5 \%$ & $32.1 \%$ & $32.3 \%$ & $32.6 \%$ & \\
\hline Which part of the nose & The lower part & 12 & 48 & 168 & 228 & 0.870 \\
\hline must be pressed to stop & (cartilage) & $37.5 \%$ & $42.9 \%$ & $42.4 \%$ & $42.2 \%$ & \\
\hline epistaxis & Upper part (bone) & 16 & 36 & 128 & 180 & \\
\hline & & $50.0 \%$ & $32.1 \%$ & $32.3 \%$ & $33.3 \%$ & \\
\hline & I do not know & 4 & 28 & 100 & 132 & \\
\hline & & $12.5 \%$ & $25.0 \%$ & $25.3 \%$ & $24.4 \%$ & \\
\hline What is the best position & Lie down with your feet & 8 & 8 & 12 & 28 & 0.029 \\
\hline to stop epistaxis & up & $25.0 \%$ & $7.1 \%$ & $3.0 \%$ & $5.2 \%$ & \\
\hline & Tilt the head forward & 8 & 32 & 204 & 244 & \\
\hline & & $25.0 \%$ & $28.6 \%$ & $51.5 \%$ & $45.2 \%$ & \\
\hline & Tilt the head back & 8 & 48 & 140 & 196 & \\
\hline & & $25.0 \%$ & $42.9 \%$ & $35.4 \%$ & $36.3 \%$ & \\
\hline & I do not know & 8 & 24 & 40 & 72 & \\
\hline & & $25.0 \%$ & $21.4 \%$ & $10.1 \%$ & $13.3 \%$ & \\
\hline & No & 8 & 44 & 84 & 136 & 0.388 \\
\hline
\end{tabular}




\begin{tabular}{|c|c|c|c|c|c|c|}
\hline \multirow[t]{2}{*}{ Questions } & \multirow[t]{2}{*}{ Responses } & \multicolumn{3}{|c|}{ Educational level } & \multirow{2}{*}{$\begin{array}{r}\text { Total } \\
(\mathrm{N}=\mathbf{5 4 0})\end{array}$} & \multirow{7}{*}{$\begin{array}{c}P \\
\text { value }\end{array}$} \\
\hline & & $\begin{array}{l}\text { Less than } \\
\text { econdary } \\
(\mathbf{n}=32)\end{array}$ & $\begin{array}{l}\text { Secondary } \\
(n=112)\end{array}$ & $\begin{array}{l}\text { University } \\
\text { or more } \\
(n=396)\end{array}$ & & \\
\hline \multirow{5}{*}{$\begin{array}{l}\text { Pressing on the nose } \\
\text { stops epistaxis }\end{array}$} & & $25.0 \%$ & $39.3 \%$ & $21.2 \%$ & $25.2 \%$ & \\
\hline & \multirow[t]{2}{*}{ I do not know } & 4 & 16 & 84 & 104 & \\
\hline & & $12.5 \%$ & $14.3 \%$ & $21.2 \%$ & $19.3 \%$ & \\
\hline & \multirow[t]{2}{*}{ Yeah } & 20 & 52 & 228 & $\mathbf{3 0 0}$ & \\
\hline & & $62.5 \%$ & $46.4 \%$ & $57.6 \%$ & $55.6 \%$ & \\
\hline \multirow{6}{*}{$\begin{array}{c}\text { Excess Nose } \\
\text { Manipulation causes } \\
\text { Epistaxis }\end{array}$} & \multirow[t]{2}{*}{ No } & $\mathbf{0}$ & 4 & 88 & 92 & \multirow[t]{6}{*}{0.016} \\
\hline & & $.0 \%$ & $3.6 \%$ & $22.2 \%$ & $\mathbf{1 7 . 0 \%}$ & \\
\hline & \multirow[t]{2}{*}{ I do not know } & 8 & 4 & 64 & 76 & \\
\hline & & $25.0 \%$ & $3.6 \%$ & $16.2 \%$ & $14.1 \%$ & \\
\hline & \multirow[t]{2}{*}{ Yeah } & 24 & 104 & 244 & 372 & \\
\hline & & $75.0 \%$ & $92.9 \%$ & $61.6 \%$ & $68.9 \%$ & \\
\hline \multirow{6}{*}{$\begin{array}{l}\text { Some medication can } \\
\text { cause epistaxis }\end{array}$} & \multirow[t]{2}{*}{ No } & $\mathbf{0}$ & 32 & 56 & 88 & \multirow[t]{6}{*}{0.089} \\
\hline & & $.0 \%$ & $28.6 \%$ & $14.1 \%$ & $16.3 \%$ & \\
\hline & \multirow[t]{2}{*}{ I do not know } & 20 & 52 & 152 & 224 & \\
\hline & & $62.5 \%$ & $46.4 \%$ & $38.4 \%$ & $41.5 \%$ & \\
\hline & \multirow[t]{2}{*}{ Yeah } & 12 & 28 & 188 & 228 & \\
\hline & & $37.5 \%$ & $25.0 \%$ & $47.5 \%$ & $42.2 \%$ & \\
\hline \multirow{6}{*}{$\begin{array}{l}\text { Chronic diseases are risk } \\
\text { factors }\end{array}$} & \multirow[t]{2}{*}{ No } & 4 & 24 & 76 & 104 & \multirow[t]{6}{*}{0.264} \\
\hline & & $12.5 \%$ & $21.4 \%$ & $19.2 \%$ & $19.3 \%$ & \\
\hline & \multirow[t]{2}{*}{ I do not know } & 24 & 36 & 188 & 248 & \\
\hline & & $75.0 \%$ & $32.1 \%$ & $47.5 \%$ & $45.9 \%$ & \\
\hline & \multirow[t]{2}{*}{ Yeah } & 4 & 52 & 132 & 188 & \\
\hline & & $12.5 \%$ & $46.4 \%$ & $33.3 \%$ & $34.8 \%$ & \\
\hline
\end{tabular}

\section{DISCUSSION}

Epistaxis is a common symptom in otorhinolaryngology practice, with most people suffering one or more episodes in their lifetime. Epistaxis means bleeding from the nostril, nasal cavity or nasopharynx. It occurs due to the bursting of a blood vessel in the nose. Although epistaxis in most cases is relatively minor and usually manageable at home, sometimes, it can be dramatic and can be a life-threatening problem. The incidence of epistaxis was reported to be ranged from $10 \%$ to $60 \%$ of individuals ${ }^{[4]} 6 \%$ of the individuals were admitted to medical treatment to control the nasal hemorrhage ${ }^{[11]}$.

Some authors portray epistaxis as a disease of the young ${ }^{[12,13]}$ whereas others have noted epistaxis to be more common in the elderly ${ }^{[14]}$. Epistaxis may occur due to various underlying causes with a significant morbidity and even mortality in rare cases. This across sectional study was conducted among 540 participants, KSA. Among 540 participants we found $45.2 \%$ of them had epistaxis. Our result was less than another study conducted among sample of 1,114 participants which found that $49 \%$ of the participants were epistaxis patients ${ }^{[15]}$. However, in Tanzania a cross-sectional, hospital based study was done to 427 patients reported that prevalence of epistaxis was $23.4 \%{ }^{[16]}$. Study done by Kishve $\boldsymbol{e t}$ al. showed the prevalence of epistaxis among pediatric patients with ear nose and throat

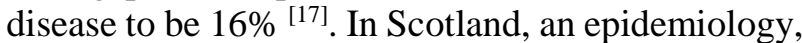
hospital based study conducted between 1995 and 2004 reported that epistaxis accounted for $33 \%$ of all ENT emergency admissions during the study period [18]. Another study conducted among 392 participants found that $27 \%$ of the participants were suffering from nasal bleeding (Epistaxis) ${ }^{[19]}$. Another study was conducted among 300 medical students from all over the kingdom of Saudi Arabia by using questionnaires reported high epistaxis prevalence, $85.67 \%$ of the participants had epistaxis at least once in their life ${ }^{[20]}$.

Regarding to knowledge of the causes of epistaxis and how to deal with epistaxis cases. Among participants, $34.8 \%$ knew that chronic diseases cause epistaxis, $42.2 \%$ stated that drugs cause epistaxis and $68.9 \%$ knew that excess nose manipulation cause epistaxis. More than half of participants $(55.6 \%)$ knew that pressing on the nose stops epistaxis. $61.5 \%$ of cases reported breath from the mouth as usual as a method for breathing during epistaxis. The majority $(81.5 \%)$ prefer going to the hospital if the epistaxis continue. 
ENT physicians reported as a specialist who can treat epistaxis by $67.4 \%$ of cases. Another study found that $67.4 \%$ of participants knew about epistaxis management, while $32.6 \%$ did not know. There were 5 questions to investigate the knowledge of individuals; the first asked if the chronic disease was a risk factor, most of the participants $46.3 \%$ did not know. In contrast to our results this study found that the majority of participants $(41.4 \%)$ did not know if some medicinal were risk factors.

There were $71.2 \%$ thought that dealing with nose was a risk factor and $77.9 \%$ thought that environmental factors may be a cause of epistaxis and high percent $64.5 \%$ thought that compressing nose was beneficial to stop bleeding. Among participants, $84.8 \%$ stated that the mechanism of breathing during epistaxis should be by mouth to stop epistaxis, $89 \%$ of individuals found that referring to the hospital was the solution to stop bleeding if the bleeding did not stop ${ }^{[21]}$. In contrast to our results, the same study reported that $70.6 \%$ of participants suggested that any medical person know epistaxis management can perform first aid followed by $29.4 \%$ for ENT physician ${ }^{[21]}$.

Another study was conducted among 600 participants reported that majority of them did not know either chronic diseases or medication are risk factors for epistaxis $(82 \%)$ and $(74 \%)$ respectively. Two-thirds of participants $(77.7 \%)$ stated compression on the nose will stop bleeding ${ }^{[22]}$.

In addition, more than $90 \%$ of participants will go to hospital if bleeding did not stop and $73.3 \%$ do not know who is managing the epistaxis, while $26.7 \%$ chose by ENT doctors ${ }^{[22]}$. As regards best position to stop epistaxis, $45.2 \%$ stated tilt the head forward, $36.3 \%$ reported tilt the head back and only $5.2 \%$ lying down with your feet up as a best position to stop epistaxis. Similar to our results, another study reported that the majority $56.9 \%$ of the participants thought that leaning the head forward is the best body position to stop nose bleeding followed by leaning the head backward in $36.5 \%$ participants, lying on the back in $5.1 \%$ participants and lying on the abdomen in only $1.5 \%$ participants ${ }^{[19]}$. Nisreen et al. ${ }^{[23]}$ found that $80.6 \%$ of the respondents in his study knew the correct position which a patient with epistaxis should be placed.

This was higher than reported by Mugwe $\boldsymbol{e t}$ al. [24] who found that $60 \%$ of respondents knew the correct position. However, Strachan and Strachan D, England ${ }^{[25]}$ reported that only $36 \%$ knew the correct position. In contrast to our results another study reported that $53 \%$ of the participants believed that lying on backward is the optimal position to stop bleeding ${ }^{[22]}$.
First aid measures with adequate knowledge required to manage acute epistaxis without hospital facilities are essential but poorly known, even though the prevalence of epistaxis is high [26, 27]. Although, the majority of cases $89.6 \%$ stated that first aid measures are important, $85.9 \%$ of them knew that there was no enough awareness of the public about epistaxis first aid measures. Similar to our results, another study reported that $92.2 \%$ of cases stated that first aid management against epistaxis was important and $76.9 \%$ thought that there was low awareness about first aid management against epistaxis [21]. Regarding to part of nose should be pressed on to stop epistaxis. Our study reported the lower part (Cartilage) by $42.2 \%$, upper part (bone) $33.3 \%$ and $24.4 \%$ did not know the correct part. Another study reported; $53.3 \%$ of cases stated that cartilage was the part comprised to stop epistaxis and $46.7 \%$ stated bony part ${ }^{[22]}$.

Also, the study conducted by Mugwe et al. ${ }^{[24]}$ showed only $38.1 \%$ correctly demonstrated pinching the nose at the cartilaginous part. According to the relation between knowledge of the causes of epistaxis and how to deal with epistaxis cases and having epistaxis attacks we found that there were no significant correlations between having epistaxis attacks and physician who can treat epistaxis, no enough awareness of the public about epistaxis first aid measures, importance of first aid measures, knowing method of breathing during epistaxis, the action should be done if the epistaxis continues, knowing which part of the nose must be pressed to stop epistaxis, the best position to stop epistaxis and knowing that pressing on the nose can stop epistaxis.

Also, there was no relation between it and drugs and chronic diseases as a cause epistaxis $(\mathrm{p}>$ $0.05)$. However, we found that there were significant correlations between epistaxis attack and nose manipulation $(\mathrm{p}=0.02)$. In our study, there was no relation between knowledge of the causes of epistaxis and how to deal with epistaxis cases and educational level. Our study reported no significant correlations between educational level and different knowledge of the causes and how to deal with epistaxis case ( $p>0.05)$.

However, we found that there was a significant correlation with the action should be done if the epistaxis continues ( $\mathrm{p}=0.001)$, the best position to stop epistaxis $(\mathrm{p}=0.02)$ and nose manipulation as a cause of epistaxis $(\mathrm{p}=0.01)$.

\section{CONCLUSION}

Our study concluded that there were $45.2 \%$ of cases had epistaxis previously. The majority $45.9 \%$ did not know that chronic diseases cause epistaxis, 
but $42.4 \%$ of them knew that drugs cause epistaxis. The majority (89.6\%) knew that first aid measures were important.

However, high percentage knew that there was no enough awareness of the public about epistaxis first aid measures. ENT physicians was reported by $67.4 \%$ as specialist who can treat epistaxis.

\section{REFERENCES}

1. Douglas $R$ and Wormald $P$ (2007): Update on epistaxis. Current Opinion in Oto-laryngology and Head and Neck Surgery, 15:180-183.

2. Albouq N, Aljeraisi T, Arabi S, Neyaz H, Alkhurassi $H$ and Alim B (2017): Knowledge and attitude regarding first aid management of epistaxis. EJPMR., 4(2):264-267.

3. Lang F (2009): Encyclopedia of Molecular Mechanisms of Disease. Springer, Berlin.

4. Varshney S et al. (2005): Epistaxis: a retrospective clinical study. Indian Journal of Otolaryngology and Head and Neck Surgery, 57(2):125-130.

5. Faistauer M, Faistauer A, Grossi RS and Roithmann R (2009): Clinical outcome of patients with epistaxis treated with nasal packing after hospital discharge. Braz. J. Otorhinolaryngol., 75(6):857-865.

6. Verma RK, Bakshi J and Panda N (2012): Ectopic intranasal tooth: an unusual cause of epistaxis in a child. Ear, Nose and Throat Journal, 91(6): 242-244.

7. Whymark AD, Crampsey DP, Fraser L, Moore P, Williams C et al. (2008): Childhood epistaxis and nasal colonization with Staphylococcus aureus. Otolaryngology Head Neck Surg., 138(3):307-310.

8. American Heart Association (2005): First Aid Science Advisory Board Evidence Evaluation Conference, hosted by the American Heart Association and the American Red Cross in Dallas, Texas. (2005). URL: http://circ.ahajournals.org/content/112/22_suppl/III115.full (Jan.23-24.2005).

9. Frazee TA and Hauser MS (2000): Nonsurgical management of epistaxis. J. Oral Maxillofac. Surg., 58:419-422.

10. Strachan D and England J (1998): First aid treatment of epistaxis -confirmation of widespread ignorance. Postgrad. Med. J., 74: 113-114.

11. Mcgarry GW and Moulton C (1993): The first aid management of epistaxis by accident and emergency department staff. Archives of Emergency Medicine, 10: 298-300.

12. Okafor BC (1984): Epistaxis: a clinical study of 540 cases. Ear Nose Throat J., 63:153-159.

13. Mgbor NC (2004): Epistaxis in Enugu: a 9 year review. Nig. J. of Otolaryngology, 1(2):11-14.
14. Pallin DJ, Chng Y, Mckay MP, Emond JA, Pelletier AJ and Camargo CA (2005): Epidemiology of epistaxis in US emergency departments, $1992-2001$. Annals of Emergency Medicine, 46(1):77-81.

15. Alhaddad MS, Almulhim K, Mubarak IAS, Alotaibi N, Hussain MAS and Alyahya KA (2017): Prevalence of epistaxis in Saudi population. Int. J.Sci. Stud., 5(9):96-99.

16. Abraham $\mathrm{Z}$ et al. (2017): Otorhinolaryngology services at Muhimbili National Hospital and Muhimbili Orthopedic Institute, Dar El Salaam, Tanzania. Medical Journal of Zambia, 44.3:184-192.

17. Kishve SP, Kumar N, Kishve PS, Aarif SM and Kalakoti P (2010): Ear, nose and throat disorders in pediatric patients at a rural hospital in India. Australasian Medical Journal, 3(12): 786-790.

18. Walker TW, Macfarlane TV and Mc Garry GW (2007): The epidemiology and chronobiology of epistaxis: an investigation of Scottish hospital admissions 1995 - 2004. Clinical Otolaryngology, 32(5):361-366.

19. Aljuaid F et al. (2018): Knowledge about the prevalence and attitude of patients experiencing epistaxis in Saudi Arabia. Egyptian Journal of Hospital Medicine, 73(6):6905-6909.

20. Alsaad S et al. (2018): Awareness about first aid management of epistaxis among medical students in Kingdom of Saudi Arabia. The Egyptian Journal of Hospital Medicine, 72(1):3726-3732.

21. Khaled A et al. (2017): Assessment of knowledge attitude and practice of epistaxis in Saudi population. The Egyptian Journal of Hospital Medicine, 69(6):26752679.

22. Al Radhwan H et al. (2018): Evaluation of knowledge attitude and practice of general population towards epistaxis in Saudi Arabia. American Journal of Pharmaceutical Sciences, 5:16948-16951.

23. Nisreen b, Talal J, Sahal A et al. (2017): Knowledge and attitude regarding first aid management of epistaxis among medical specialties students in Al-Madinah, Kingdom of Saudi Arabia. International Journal of Scientific and Engineering Research, 4(2): 264-267.

24. Mugwe P, Kamau $K$ and Nyambaka $O$ (2014): Knowledge, attitude and practice in first aid management of epistaxis by accident and emergency clinical staff at Kenyatta National Hospital. East and Central African Journal of Surgery, 19(1):17-21.

25. Strachan D and England J (1998): First-aid treatment of epistaxis- confirmation of widespread ignorance. Postgrad. Med. J., 74(868):113-117.

26. Khan A, Shaikh S, Shuaib F, Sattar A, Samani SA, Shabbir Q et al. (2010): Knowledge attitude and practices of undergraduate students regarding first aid measures. J. Pak. Med. Assoc., 60: 68-72.

27. Tekian A (2002): Have newly graduated physicians mastered essential clinical skills? Medical Education, 36(5):406-413. 